\title{
Decision Support for Gasoline Tanker Logistics with BPsim.DSS
}

\author{
K. Aksyonov, E. Bykov, O. Aksyonova, N. Goncharova, A. Nevolina \\ Depart ment of Information Technology \\ Ural Federal University \\ Russia
}

\begin{abstract}
The paper describes deployment experience of a decision support system for planning fuel supplies within a network of gas stations, which is based on simulation, multi-agent and expert modeling. Authors focus on various methods used in decision support system BPsim.DSS. Mainly the system is used by logistical management and planning departments. The system implement such features as forecasting next day fuel sales, searching for effective fuel supply plan, planning trips for each fuel tanker. Simulation model estimates fuel sales. Planning is implemented in BPsim.MSN on the basis of logical output visual machine, based on UML diagrams and T-SQL language scripts. Testing results prove effectiveness of decisions: sales volume can be increased by optimizing usage of fuel tankers.
\end{abstract}

\section{Keywords-agents; decision support systems; logistics}

\section{INTRODUCTION}

Transportation planning is based on scheduling theory and transportation graphs, built upon systematization of contracts, available orders, and study of cargo flows. Schedules and graphs must provide

Satisfaction of requirements of the maximum number of transportation customers,

Minimization of time costs for transportation,

Regularity of trans portation,

Maximization of gas sales within gas stations network,

Effective use of fuel tankers,

Interconnection with graphs and schedules of different transport types (e.g., railroad),

Minimization of empty vehicle runs.

Paper describes deployment experience of a decision support system for planning fuel supplies within a network of gas stations, which is based on simulation, multi-agent and expert modeling. We focus on modeling the of fuel distribution within a network of gas stations. We propose the agent-based network. Some researchers suggest relating to this the routing problem as the graph problem, represented by the so-called special matrices [1-3].

In our case technically we may use matrices for setting distances between different locations (gas stations, oil bases, garages). We also may apply matrices for setting physical limitations for delivery transporters, but such representations go beyond the scope of this paper.
There are four types of agents involved:

Gas stations monitor the remaining fuel and generate fuel orders,

Oil bases do the same thing in relations to larger volumes, available at the base,

Delivery transporters fulfill delivery orders,

The planning agent, which represents the dispatcher.

Initially the model may be represented in form of a full graph, but throughout model lifecycle it is dynamic, i.e. it depends on the current list of orders from gas stations and available oil bases. Thus, it may be modified. Edge (road) weights depend on the day of week, time of day and political situation of the area (this includes major exhibits, etc.). Since the problem is solved for the whole shift, the graph structure may change (new orders appear), as well as its features.

\section{REQUIREMENT S FOR INTELLIGENT PLANNING SYSTEM}

General problem of fuel distribution within a network of gas stations consists of defining a set of structures $S_{i}=\left\langle R_{i}, F_{i}, T_{i}\right\rangle$ before the beginning of working shift. Here Ri is a route for $\mathrm{i}$-th transportation, $\mathrm{Fi}$ - fuel tanker on i-th transportation, $\mathrm{Ti}$ - terms of start and end of i-th transportation, effective on the total costs criteria on the i-th transportation $C_{i}=\sum C_{i j}$, where $\mathrm{Ci} 1-$ cost of fuel transportation on the route $\mathrm{Ri}$; $\mathrm{Ci} 2$ - volume of missed profit during filling of station tank; Ci3 - fuel costs for the fuel tanker Fi and other costs of $i$-th transportation.

Rational design of transportation distribution system of an enterprise is based on elements of linear programming, scheduling theory, simulation, multi-agent, and expert modeling. In order to compile an effective transportation plan authors offer the method of sequential improvement of initial transportation scheme, which is based on linear programming theory together with simulation model of fuel tankers operation. The method consists of the following stages:

Generation of information structures, corresponding to orders from gas stations, which is in turn based on the current fill ratio of station fuel tanks;

Defining supplier and delivery route for each order,

Assignment of a fuel tanker to each order, determination of terms for delivery,

Manual correction of the plan by an expert. Checking and correction of the plan on the simulation model. 
The proposed method of combined order distributing between suppliers and fuel tankers allows a complete solution for designing transportation plan. The method also provides capability to select the most applicable option of fuel tankers assignment, depending on their condition, as well as redesigning the scheme in case of emergencies.

\section{MATHEMATICAL MODEL}

The model is based on proven efficient apparatus of multiagent resource conversion processes. Multi-agent resource conversion process structure is presented on Figure 1. Resource conversion process (RCP) is the process of an input conversion (resources necessary for process execution) into output (products - outcomes of process execution). The main objects of discrete Multi-agent RCP are presented on Figure 1: operations $(O p)$, resources (Res), control commands $(U)$, conversion devices (Mech), processes $(P R)$, sources (Sender) and resource receivers (Receiver), junctions (Junction), parameters $(P)$, agents (Agent). Process parameters are set by the object characteristics function. Relations between resources and conversion device are set by link object (Relation). The agents existence resumes availability of the situations (Situation) and decisions (action plan) (Decision).

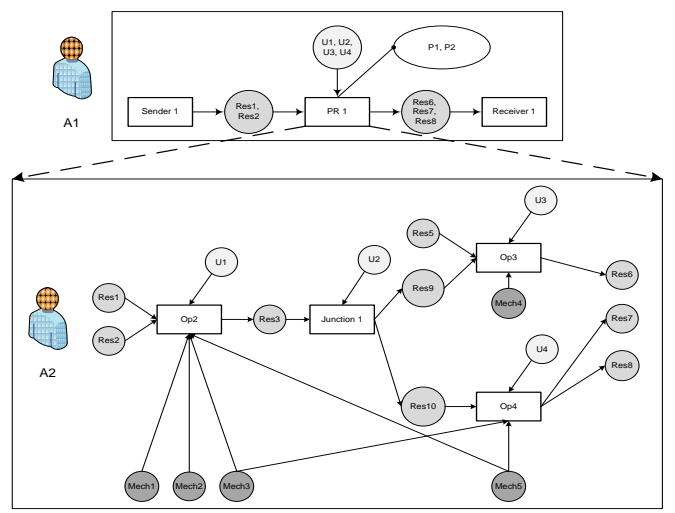

FIGURE I. MULTI-AGENT RESOURCE CONVERSION PROCESS MODEL ST RUCTURE.

They further extended concept of agents involved in the model. Multi-agent resource conversion process agent may have hybrid nature and contain two components:

Intelligent (production rules and/or frame-based expert system access)

Reactive (agent activity is defined on UML activity diagram).

In accordance with InteRRaP architecture common concept, multi-agent RCP agent model is represented in four levels.

Stages 1-4 are implemented on the basis of logical output frame machine in planning sub-system (presented in decision support system BPsim.DSS), 5th stage is implemented in simulation multi-agent model (presented in dynamic situations modeling system BPsim.MAS).

Agents of the multi-agent system correspond to transportation vehicles, logistical locations (for loading and unloading), gas stations, rest areas. The designed automated control system will solve the following problems:
Optimize use ratio for transportation vehicles (cargo distribution between vehicles, considering delivery terms),

Planning optimal route, considering traffic capacity of the highways, availability of fueling stations, hotels, food areas,

Scheduling drivers' work, Monitoring vehicle and cargo location.

\section{SOFTWARE IMPLEMENT ATION AND APPLICATION OF INTELLIGENT PLANNING SYSTEM}

Decision support system is implemented on the basis of BPsim.DSS software suite $[4,5]$. BPsim.DSS targets modeling complex business systems. Architecture of BPsim.DSS relies on the principles of three-tier hierarchical architecture InteRRaP [6]. Interaction of intelligent system modules is presented on Figure 2.

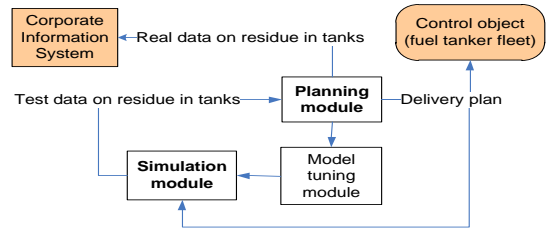

FIGURE II. INTERACTION OF PLANNING SYSTEM.

At present the intelligent planning system is under development. It is going to be deployed at logistical department of gas supplying company, located in Ekaterinburg, Russia, a major city in authors' area. The gas stations network of the supplier consists of 24 gas stations.

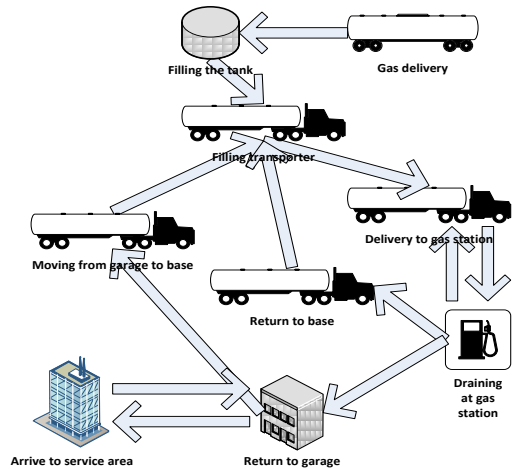

FIGURE III. GAS ST ATIONS SERVICE PROCESS.

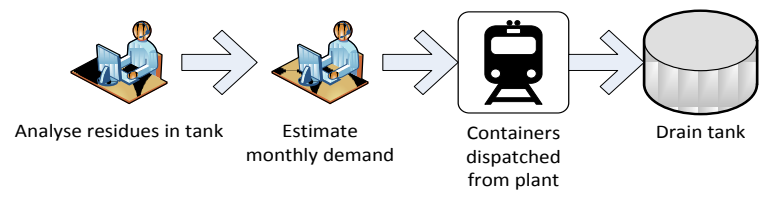

FIGURE IV. DELIVERING FUEL TO THE BASE.

In order to improve speed of simulation models, including intelligent agents, the production knowledge base rules were separated into 2 types: 1. Global condition rules, and 2. General rules. If an intelligent agent contains both rule types, then the global conditions are checked in the first place, and in case a condition is fulfilled, the full knowledge base scan is started (including general rules). If global rules are not contained within an agent, then the search is carried out on the 
whole knowledge base. Such separation of rules into two times allowed great improvement in speed (approximately 5 times faster).

Initially a logistics specialist sets initial values. They include date of the plan, fuel tankers shift start time, distribution strategy for various fuel types. Initial data also includes residues at gas stations and technical condition of tanker fleet. After finish of algorith m operation, users have an opportunity to modify the plan. Next the plan is exported into simulation module and is corrected depending on simulation results. Simulation model also monitors residues on each gas station and fuel usage dynamics.

A regional gasoline company "SKON" owns 22 gas stations. Fuel to these stations is delivered by 12 fuel tankers. The process of servicing gas stations is visually presented on Figures 3, 4.

Each gas station operates 24/7. Customers arrive after certain time intervals and purchase fuel. When fuel level at the station reaches specific minimum level, additional fuel delivery is requested.In order to have no delays in fuel supply, fuel residues in the base tanks should be timely analyzed. When fuel level in one of the tanks at the base reaches minimum level, fuel is ordered to the base. The train to the base arrives after 7 days. Fuel is drained from the train to tanks for 40 minutes.

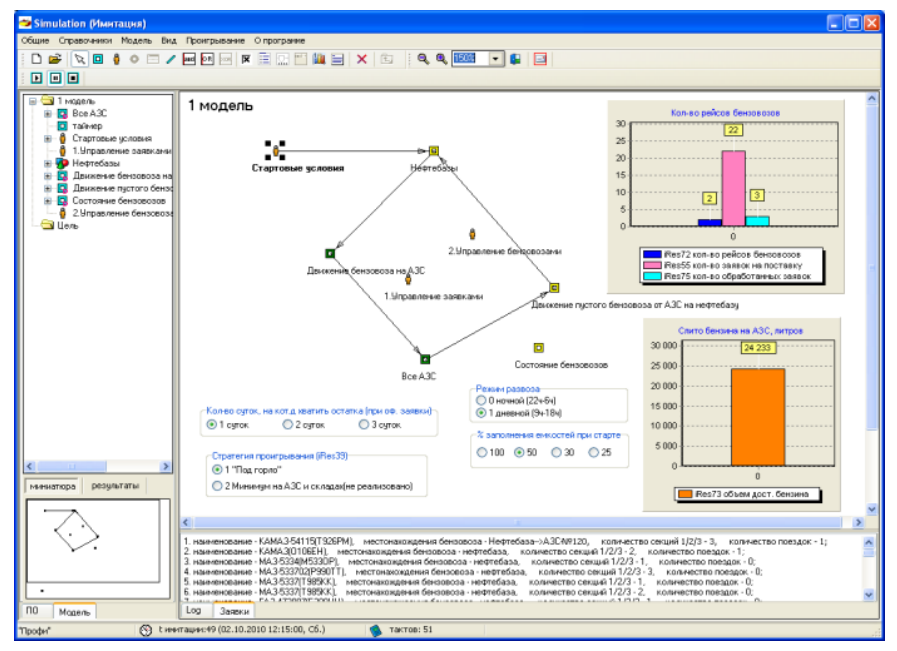

FIGURE V. TOPMOST MODEL LEVEL.

Figure 5 presents the topmost level of the model. Since the system was applied at a Russian company, all model objects are in Russian, though their specific names are not important for understanding. Here we present the main interface with key model object, including initial conditions, gasoline farms (bases), control processes, and gas stations. Most objects are further decomposed, thus having comprehensive structure.

Some model blocks are decomposed and their further definition is presented on the next model levels. The total number of fuel tanks at all gas stations is 85 .

Agents of "All gas stations" node simulate fuel consumption from each of the tanks. Depending on time of day consumption changes, decreasing at night (11pm-7am) and increasing at day (7am-11pm). Fuel level is displayed on diagrams, presented on Figures 6-7. The first one shows current levels at the tanks, the latter one - progress of fuel level over time.

Generated transacts are put into queue, after that they are distributed between a vailable fuel tankers by control units. In case of multiple transacts for a single gas station, the single tanker is used.

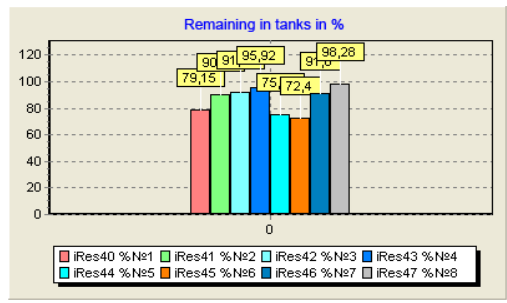

FIGURE VI. CURRENT FUEL LEVEL IN GAS ST ATION TANKS.

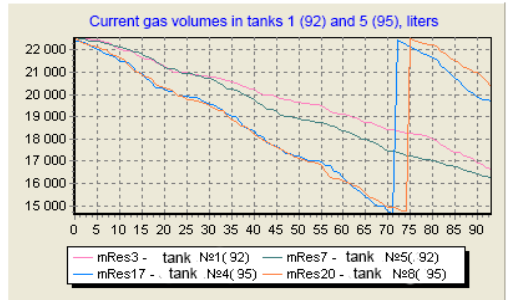

FIGURE VII. FUEL LEVEL DYNAMICS FOR THETANKS OF A SPECIFIC GAS ST AT ION. CONCLUSION

The problem of deliveries to a network of gas stations and its software implementation is solved with aid of hybrid approach, with application of linear programming method (modified transport algorithm), heuristic planning algorith $\mathrm{m}$ and multi-agent simulation modeling.

\section{ACKNOWLEDGEMENT}

Research is conducted under the terms of contract № 02.G25.31.0055 (project 2012-218-03-167) under financial support of Ministry of Education and Science of Russian Federation.

\section{REFERENCES}

[1] Respondek J.S., Numerical recipes for the high efficient inverse of the confluent Vandermonde matrices, Applied Mathematics and Computation, Volume 218, Issue 5, pp. 2044-2054, 2011.

[2] Bollobas B., Modern Graph Theory, Springer 1998

[3] [3] Aceto L., The matrices of Pascal and other greats, Am. Math. Mon. 108 (3) (2001) 232245.

[4] Aksyonov K.A., Sholina I.I. \& Sufrygina E.M. 2009. Multi-agent resource conversion process object-oriented simulation and decision support system development and application, Scientific and technical bulletin, Vol. 3 (80), Informatics. Telecommunication. Control, St.Petersburg, pp.87-96.

[5] Aksyonov, K.A. 2011. Theory and practice of decision support tools, Germany, Saarbrucken: LAP LAMBERT Academic Publishing GmbH \& Co. KG. ISBN 978-3-8465-0782-7.

[6] Muller J.P. \& M.Pischel. 1993. The Agent Architecture InteRRaP: Concept and Application, German Research Center for Artificial Intelligence (DFKI) G. Eason, B. Noble, and I. N. Sneddon, "On certain integrals of Lipschitz-Hankel type involving products of Bessel functions," Phil. Trans. Roy. Soc. London, vol. A247, pp. 529-551, April 1955. 